\title{
Cataract in Leprosy: A Biochemical Approach*
}

\author{
K. PRABHAKARAN \\ U.S.P.H.S. Hospital, Carville, Louisiana 70721, U.S.A.
}

\begin{abstract}
The possible rôle of phenoloxidase of Mycobacterium leprae in producing cataract in leprosy is discussed. It is suggested that diethyldithiocarbamate, a potent inhibitor of the enzyme, could be of value as a therapeutic agent.
\end{abstract}

Host and tissue preferences of pathogenic bacteria have been attributed to differences in nutritional conditions in different hosts and in different tissues within the same host. A case in point is the invasion by brucellae of certain tissues in pregnant animals, where erythritol is present (Smith, 1968). The human leprosy bacilli multiply at sites in the human body (e.g. skin and peripheral nerve) where metabolism of 3,4-dihydroxyphenylalanine (dopa) or its derivatives is important. Dopa is a precursor of melanin pigment, and of norepinephrine which is a neurotransmitter. In vitro studies have shown that Mycobacterium leprae rapidly oxidizes dopa and its analogues (Prabhakaran et al., 1969).

Involvement of the eye is common in advanced cases of leprosy. Besides the nerves supplying different areas of the eye, various parts of the organ are invaded by the bacilli. Large numbers of Myco. leprae have been found in the cornea, iris, and the ciliary body. The ciliary body is gradually destroyed-a major cause of blindness in leprosy. Cataracts commonly develop and sometimes vitreous opacities (Choyce, 1964). It is interesting that dopa has been shown to be present in the iris and ciliary body (Pirie, 1968). Various lines of evidence suggest that the metabolism of dopa or its derivatives may be important in the multiplication of Myco. leprae.

The enzyme that oxidizes dopa or tyrosine to pigmented products is known as tyrosinase or phenoloxidase. It has been suggested that cataract formation in man is due to the action of quinones formed from dopa or tyrosine by the activity of tyrosinase (Srivastava and Nath, 1968). Homogenates of senile cataractous lenses were found to have tyrosinase activity; this activity could be inhibited by the addition of reducing agents (ascorbic acid or glutathione) to the homogenates. Normal lenses contained no tyrosinase. However, when inhibitory metabolites were removed by dialysis, homogenates of normal lenses also showed tyrosinase. It is likely that in old age a decrease in reducing agents activates tyrosinase of the

* Received for publication 10 December, 1970. 
lens. The quinones produced in the oxidation of dopa or tyrosine might form compounds with the lens proteins, leading to the cataractous condition. In the presence of tyrosinase the quinones also oxidize -SH groups of proteins to produce insoluble -S-S- proteins which cause opalescence in the cataractous lens (Srivastava and Nath, 1968).

Human cataractous lenses vary in colour from pale yellow to deep brown. The brown colour is associated with an insoluble protein in the lens. Cataractous lenses of naphthalene-fed rabbits contain brown proteins; these are compounds of lens proteins with naphthoquinone (Pirie, 1968). Dopa, as well as tyrosinase, occurs in areas of the eye such as the iris and ciliary body. It is likely that the brown colour of the human cataractous lens is due to compounds of lens proteins with quinones which are formed from tyrosine or dopa by tyrosinase. When homogenates of cattle lenses were incubated with a mixture of tyrosine, dopa and tyrosinase, the lens proteins gradually developed yellow and brown colours, and became black on prolonged incubation. Concomitantly, there was a loss of thiol groups from the proteins (Pirie, 1968).

Myco. leprae was found to oxidize dopa and several other phenolic compounds in vitro (Prabhakaran et al., 1969). As a result, when leprosy bacilli invade the eye they could oxidize the dopa present, and so give rise to quinones. These quinones might interact with the proteins of the lens, leading to a cataractous condition. Thus, in leprosy there are 2 factors contributing to cataract formation: (i) the tyrosinase of the lens, and (ii) the tyrosinase of Myco. leprae.

It has been shown that tyrosinase activity present in homogenates of senile cataractous lenses could be inhibited by the addition of reducing agents like ascorbic acid and glutathione (Srivastava and Nath, 1968). As such, the administration of high levels of ascorbic acid might be expected to help alleviate the cataract in leprosy patients resulting from the tyrosinase of the lens. However, in vitro experiments have shown that the phenoloxidase of Myco. leprae is different from the tyrosinase of mammalian or plant origin, in the effect of reducing agents on the enzyme (author's unpublished results). The formation of quinone from dopa by the latter 2 enzymes was completely suppressed in the presence of ascorbic acid and glutathione; on the other hand, reducing agents had little effect on the oxidation of dopa to quinone by Myco. leprae. Therefore, it is likely that the administration of ascorbic acid may not prove to be completely effective in combating the cataract which directly results from invasion of the eye by Myco. leprae.

In this situation, a search for inhibitors which suppress tyrosinase of Myco. leprae is important. Non-toxic compounds which completely suppress tyrosinase of the leprosy bacilli would prevent the formation of quinone which causes the cataract. In our experiments, the most effective inhibitor of tyrosinase from different sources was diethyldithiocarbamate (DDC) (Prabhakaran et al., 1969). This compound produced total inhibition of the enzyme in Myco. leprae. DDC has been successfully used in the treatment of Wilson's disease (hepato-lenticular degeneration) and in the case described improvement of the eyesight was also reported (Sunderman, Jr. et al., 1963). DDC penetrates even intact Myco. leprae and suppresses its tyrosinase activity. A sulphone drug tested was less effective (Prabhakaran et al., 1969). Moreover, although sulphone treatment sometimes arrests the progress of ocular leprosy, a cure rarely takes place. The above considerations indicate that DDC might be effective in preventing quinoneformation from dopa by Myco. leprae and thus help alleviate the cataractous 
condition so common in leprosy. Our preliminary results on multiplication of Myco. leprae in the mouse footpad (to be published) also indicate that DDC, besides preventing quinone formation from dopa, might also lead to killing of the leprosy organisms.

\section{Acknowledgements}

This investigation was supported by the United States-Japan Cooperative Medical Science Program administered by the National Institute of Allergy and Infectious Diseases of the National Institutes of Health, Department of Health, Education, and Welfare. I thank Dr. Margaret B. Brand, Chief, Ophthalmology Department, USPHS Hospital, Carville, for her comments and helpful suggestions.

\section{References}

Choyce, D. P. (1964). The eyes in leprosy. In "Leprosy in Theory and Practice", 2nd ed., p. 310 . Bristol: John Wright \& Sons. Baltimore: The Williams \& Wilkins Co.

Pirie, A. (1968). Reaction of tyrosine oxidation products with proteins of the lens. Biochem. $J$. 109,301 .

Prabhakaran, K., Harris, E. B. and Kirchheimer, W.F. (1969). Effect of inhibitors on phenoloxidase of Mycobacterium leprae. J. Bact. 100, 935.

Smith, H. (1968). Biochemical challenge of microbial pathogenicity. Bact. Rev. 32, 164.

Srivastava, S. K. and Nath, R. (1968). Appearance of $o$-diphenol oxidase in senile cataractous lens. Indian J. Biochem. 5, 145.

Sunderman, Jr., F. W., White, J. C., Sunderman, F. W. and Lucyszyn, G. W. (1963). Metabolic balance studies in hepato-lenticular degeneration treated with diethyldithiocarbamate. Am. J. Med. 34, 875 . 